\title{
PROOF OF A CONJECTURE OF KOSTANT
}

\author{
DRAGOMIR Ž. ĐOKOVIĆ
}

\begin{abstract}
Let $\mathfrak{g}_{0}=\mathfrak{k}_{0}+\mathfrak{p}_{0}$ be a Cartan decomposition of a semisimple real Lie algebra and $\mathfrak{g}=\mathfrak{k}+\mathfrak{p}$ its complexification. Denote by $G$ the adjoint group of $\mathfrak{g}$ and by $G_{0}, K, K_{0}$ the connected subgroups of $G$ with respective Lie algebras $\mathfrak{g}_{0}, \mathfrak{k}, \mathfrak{k}_{0}$. A conjecture of Kostant asserts that there is a bijection between the $G_{0}$-conjugacy classes of nilpotent elements in $\mathfrak{g}_{0}$ and the $K$-orbits of nilpotent elements in $\mathfrak{p}$ which is given explicitly by the so-called Cayley transformation. This conjecture is proved in the paper.
\end{abstract}

1. Introduction. Let $\mathfrak{g}_{0}=\mathfrak{k}_{0}+\mathfrak{p}_{0}$ be a Cartan decomposition of a real semisimple Lie algebra and let $\mathfrak{g}=\mathfrak{k}+\mathfrak{p}$ be its complexification. Denote by $G$ the adjoint group of $\mathfrak{g}$ and by $K$ resp., $G_{0}, K_{0}$ the connected Lie subgroup of $G$ with $\mathfrak{k}$ resp., $\mathfrak{g}_{0}, \mathfrak{k}_{0}$ as its Lie algebra. We consider the adjoint action of $G$ and $G_{0}$ and their restrictions to the subgroups $K$ and $K_{0}$, respectively.

According to D. King [7] it was conjectured by B. Kostant that there is a bijection between the $G_{0}$-conjugacy classes of nilpotent elements in $\mathfrak{g}_{0}$ and the $K$-conjugacy classes of nilpotent elements in $\mathfrak{p}$ given explicitly by the so-called Cayley transformation. Of course it suffices to consider the case when $\mathfrak{g}_{0}$ is simple If $\mathfrak{g}_{0}$ is of classical type then the conjecture has been verified recently by D. King [7] using case by case considerations.

In this paper we give a proof of Kostant's conjecture (in full generality) by a completely different method. Our proof is based on Vinberg's work on the classification of nilpotent elements in graded Lie algebras.

The tables of nilpotent $K$-orbits in $\mathfrak{p}$ for exceptional simple Lie algebras $\mathfrak{g}$ will be submitted for publication elsewhere. These tables then can be considered as a classification of nilpotent $G_{0}$-conjugacy classes in $\mathfrak{g}_{0}$. When $\mathfrak{g}_{0}$ is of Cartan type EV this was accomplished by Antonyan [1], but he does not indicate which nilpotent $K$-orbits in $\mathfrak{p}$ belong to the same $G$-orbit.

I would like to thank D. King for sending me his preprint [7]. It was this preprint that prompted me to look for a direct proof of Kostant's conjecture.

2. Notations and definitions. $\mathfrak{g}_{0}$ will be a finite-dimensional real Lie algebra and $\mathfrak{g}_{0}=\mathfrak{k}_{0}+\mathfrak{p}_{0}$ its Cartan decomposition. Its complexification will be written as $\mathfrak{g}=\mathfrak{k}+\mathfrak{p}$.

$G$ denotes the adjoint group of $\mathfrak{g}$ and for each subalgebra of $\mathfrak{g}$, denoted by a german letter (possibly with a subscript), the corresponding connected Lie subgroup

Received by the editors February 5, 1986.

1980 Mathematics Subject Classification (1985 Revision). Primary 17B20, 22E60; Secondary $17 \mathrm{~B} 45$.

The support through the NSERC Grant A-5285 is gratefully acknowledged. 
of $G$ will be denoted by the corresponding uppercase italic letter (and the same subscript).

If $\mathfrak{h}$ is a Cartan subalgebra of $\mathfrak{g}$ and $\alpha$ a root of $(\mathfrak{g}, \mathfrak{h})$ then $\mathfrak{g}^{\alpha}$ will denote the corresponding root space of $\mathfrak{g}$.

Let $\mathfrak{s}=\bigoplus \mathfrak{s}_{k}$ be a $Z$-graded complex semisimple Lie algebra. Then there is a unique element $H \in \mathfrak{s}$ such that $\mathfrak{s}_{k}=\{X \in \mathfrak{s}:[H, X]=k X\}$ for all $k \in \mathbf{Z}$. Clearly $H \in \mathfrak{s}_{0}$ and we call $H$ the defining element of this $\mathbf{Z}$-graded algebra. Since $H$ determines the gradation of $\mathfrak{s}$ we shall refer to this $\mathbf{Z}$-graded Lie algebra as $(\mathfrak{s}, H)$.

If $\mathfrak{s}=\bigoplus \mathfrak{s}_{k}$ is $\mathbf{Z}$-graded then by using the canonical surjection $\mathbf{Z} \rightarrow \mathbf{Z}_{2}:=\mathbf{Z} / 2 \mathbf{Z}$ we obtain a $\mathbf{Z}_{2}$-grading of $\mathfrak{s}$ which we call the associated $\mathbf{Z}_{2}$-grading.

The algebra $\mathfrak{g}=\mathfrak{k}+\mathfrak{p}$ is a $\mathbf{Z}_{2}$-graded Lie algebra. A $\mathbf{Z}$-graded subalgebra of this $\mathbf{Z}_{2}$-graded algebra is a $\mathbf{Z}$-graded subalgebra $\mathfrak{s}=\bigoplus \mathfrak{s}_{k}$ of $\mathfrak{g}$ such that $\mathfrak{s}_{k} \subset \mathfrak{k}$ for $k$ even and $\mathfrak{s}_{k} \subset \mathfrak{p}$ for $k$ odd.

By $\theta$ we denote the automorphism of $\mathfrak{g}_{0}$ which is 1 on $\mathfrak{k}_{0}$ and -1 on $\mathfrak{p}_{0}$. We also denote by $\theta$ its extension to a complex automorphism of $\mathfrak{g}$.

By $\sigma$ we denote the conjugation of $\mathfrak{g}$ with respect to its real form $\mathfrak{g}_{0}$. If $\mathfrak{s}$ is a $\sigma$-stable subalgebra of $\mathfrak{g}$ then by $\mathfrak{s}^{\sigma}$ we denote the subalgebra of $\mathfrak{s}$ consisting of elements of $\mathfrak{s}$ fixed by $\sigma$.

A Z-graded semisimple Lie algebra $\mathfrak{g}=\bigoplus \mathfrak{s}_{k}$ is called locally flat if $\operatorname{dim} \mathfrak{s}_{0}=$ $\operatorname{dim} \mathfrak{s}_{1}$. In that case the group $S_{0}$ has precisely one open orbit in $\mathfrak{s}_{1}$ under the adjoint action and we shall refer to any element of that orbit as a generic element of $\mathfrak{s}_{1}$. For each generic element $X \in \mathfrak{s}_{1}$ the centralizer of $X$ in $S_{0}$ is finite. If this centralizer is trivial then we say that this $\mathbf{Z}$-graded algebra is flat. These definitions are due to Vinberg [11].

A subalgebra of $\mathfrak{g}$ is called regular if it is normalized by some Cartan subalgebra of $\mathfrak{g}$. A nonzero nilpotent element $X \in \mathfrak{g}$ and its $G$-conjugacy class $G \cdot X$ are said to be semiregular (in $\mathfrak{g}$ ) if $G \cdot X$ does not meet any proper regular semisimple subalgebra of $\mathfrak{g}$. Given any nonzero nilpotent element $X \in \mathfrak{g}$ there exists a regular semisimple subalgebra $\mathfrak{s}$ of $\mathfrak{g}$ such that $G \cdot X \cap \mathfrak{s}$ is nonempty and every element of this intersection is semiregular in $\mathfrak{s}$. Dynkin's classification of nilpotent $G$-conjugacy classes of $\mathfrak{g}$ is based on the classification of semiregular nilpotent classes. The semiregular nilpotent $G$-conjugacy classes are also discussed by Elkington [6].

Let $X \neq 0$ be a nilpotent element of $\mathfrak{g}$. By a theorem of Morozov there exist $H, Y \in \mathfrak{g}$ such that

$$
[X, Y]=-H, \quad[H, X]=2 X, \quad[H, Y]=-2 Y .
$$

Following Bourbaki [3] we shall call such triple $(X, H, Y)$ an $\mathfrak{s l}_{2}$-triple. (Usually one replaces the equality $[X, Y]=-H$ by $[X, Y]=H$ in the above definition but we make this departure in order to conform with the terminology of [3].)

A real Cayley triple is an $\mathfrak{s l}_{2}$-triple $(E, H, F)$ in $\mathfrak{g}_{0}$ such that $\theta(E)=F$. This implies that $\theta(F)=E, \theta(H)=-H$ and consequently $H \in \mathfrak{p}_{0}, E+F \in \mathfrak{k}_{0}$, and $E-F \in \mathfrak{p}_{0}$.

A complex Cayley triple is an $\mathfrak{s l}_{2}$-triple $(E, H, F)$ in $\mathfrak{g}$ such that $E, F \in \mathfrak{p}$ and $\sigma(E)=-F$. It follows that $\sigma(F)=-E, \sigma(H)=-H, H \in i \mathfrak{k}_{0}, E+F \in i \mathfrak{p}_{0}$, and $E-F \in \mathfrak{p}_{0}$. 
Clearly $K_{0}$ acts by adjoint action on both real and complex Cayley triples. The Cayley transfrom $c$ is a map from real to complex Cayley triples defined by

$$
c(E, H, F)=\left(\frac{1}{2}(H+i F-i E), i(E+F), \frac{1}{2}(-H+i F-i E)\right) .
$$

It is easy to check that this map is bijective and $K_{0}$-equivariant and that its inverse is given by

$$
c^{-1}(E, H, F)=\left(\frac{i}{2}(E+F-H), E-F,-\frac{i}{2}(E+F+H)\right) .
$$

Hence $c$ induces a bijection $\bar{c}$ from the set of $K_{0}$-conjugacy classes of real Cayley triples to the set of $K_{0}$-conjugacy classes of complex Cayley triples.

An $\mathfrak{s l}_{2}$-triple $(E, H, F)$ in $\mathfrak{g}$ is called normal if $E, F \in \mathfrak{p}$ and $H \in \mathfrak{k}$. They have been studied extensively by Kostant and Rallis [8].

3. Some known results. Define a map $\phi$ from the set of $K_{0}$-conjugacy classes of real Cayley triples to the set of nonzero nilpotent $G_{0}$-orbits in $\mathfrak{g}_{0}$ by assigning to the class containing the real Cayley triple $(E, H, F)$ the orbit $G_{0} \cdot E$. It is shown by King [7, Lemma 1.1] that $\phi$ is surjective.

Each $K_{0}$-conjugacy class of complex Cayley triples is contained in a unique $K$ conjugacy class of normal $\mathfrak{s l}_{2}$-triples. Hence the inclusion relation defines a map $\psi_{0}$ from the set of $K_{0}$-conjugacy classes of complex Cayley triples to the set of $K$ conjugacy classes of normal $\mathfrak{s l}_{2}$-triples. King shows that $\psi \circ \bar{c} \circ \phi^{-1}$ is a well-defined map from the set of nonzero nilpotent $G_{0}$-orbits in $\mathfrak{g}_{0}$ to the set of $K$-orbits of normal $\mathfrak{s l}_{2}$-triples (the proof is in the paragraph following Remark 1.1). He also shows that this map is injective. His proof of this fact is based on a theorem of Kostant and Rao the proof of which was published by D. Barbasch [2, Proposition 3.1]. These proofs will not be reproduced here. In the Addendum we show that $\phi$ is also injective.

Let $\psi_{1}$ be the map from the set of $K$-conjugacy classes of normal $\mathfrak{s l}_{2}$-triples to the set of nonzero nilpotent $K$-orbits in $\mathfrak{p}$ which assigns to the class containing the normal $\mathfrak{s l}_{2}$-triple $(E, H, F)$ the orbit $K \cdot E$. Kostant and Rallis [8, Proposition 4] have shown that $\psi_{1}$ is bijective.

Now we can state Kostant's conjecture: The map $\psi_{1} \circ \psi_{0} \circ \bar{c} \circ \phi^{-1}$ from nonzero nilpotent $G_{0}$-orbits in $\mathfrak{g}_{0}$ to nonzero nilpotent $K$-orbits in $\mathfrak{p}$ is bijective. Some partial results in connection with this conjecture have been obtained by L. PreissRothschild [9].

From the results stated above we know that this map is injective. This is the content of Proposition 1.2 in [7].

In order to complete the proof of the conjecture it remains to prove that $\psi_{0}$ is also surjective, i.e., that every $K$-conjugacy class of normal $\mathfrak{s l}_{2}$-triples in $\mathfrak{g}$ contains a complex Cayley triple. Equivalently, it suffices to show that the map $\psi:=\psi_{1} \circ \psi_{0}$ is surjective. That will be accomplished in $\S 5$.

The following lemma will be needed for our proof. The validity of this lemma follows from the description of nilpotent $G$-orbits in $\mathfrak{g}$, which was accomplished by Dynkin [5] (see also [6]) and the description of flat Lie algebras in [11 or 12].

LEMMA 1. Let $(E, H, F)$ be an $\mathfrak{s l}_{2}$-triple in $\mathfrak{g}$ with $E$ a semiregular nilpotent in $\mathfrak{g}$. Then $\operatorname{ad}(H / 2)$ has integer eigenvalues, the $\mathbf{Z}$-graded Lie algebra $(\mathfrak{g}, H / 2)=$ $\bigoplus_{k \in \mathbf{Z}} \mathfrak{s}_{k}$ is flat, and $E$ is a generic element of $\mathfrak{s}_{1}$. 
4. Basic lemma. For the proof of the basic lemma we need the following technical lemma.

LEMMA 2. Assume that rank $\mathfrak{k}=$ rank $\mathfrak{g}$, fix a Cartan subalgebra $\mathfrak{h}_{0}$ of $\mathfrak{k}_{0}$ and let $\mathfrak{h}=\mathfrak{h}_{0}+i \mathfrak{h}_{0}$. Let $R$ be the root system of $(\mathfrak{g}, \mathfrak{h})$ and

$$
R^{(0)}=\left\{\alpha \in R: \mathfrak{g}^{\alpha} \subset \mathfrak{k}\right\}, \quad R^{(1)}=\left\{\alpha \in R: \mathfrak{g}^{\alpha} \subset \mathfrak{p}\right\} .
$$

Then there exists a Chevalley system $\left(X_{\alpha}\right), \alpha \in R$, of $(\mathfrak{g}, \mathfrak{h})$ such that

$$
\sigma\left(X_{\alpha}\right)=(-1)^{k} X_{-\alpha}, \quad \alpha \in R^{(k)} .
$$

(For the definition of Chevalley systems see [3, Chapitre VIII, §3, no. 4, p. 84].)

Proof. Let $\left(Y_{\alpha}\right), \alpha \in R$, be any Chevalley system of $(\mathfrak{g}, \mathfrak{h})$.

The R-span of $\mathfrak{h}_{0}$ and the vectors $Y_{\alpha}+Y_{-\alpha}, i\left(Y_{\alpha}-Y_{-\alpha}\right), \alpha \in R^{(0)}$; and $i\left(Y_{\alpha}+Y_{-\alpha}\right), Y_{\alpha}-Y_{-\alpha}, \alpha \in R^{(1)}$; are a real form $\mathfrak{g}^{\#}$ of $\mathfrak{g}$ isomorphic to $\mathfrak{g}_{0}$. Choose an isomorphism $\tau: \mathfrak{g}^{\#} \rightarrow \mathfrak{g}_{0}$ such that $\tau\left(\mathfrak{h}_{0}\right)=\mathfrak{h}_{0}$ and extend $\tau$ to an automorphism of $\mathfrak{g}$. Set $X_{\alpha}=\tau\left(Y_{\alpha}\right)$. Then $\left(X_{\alpha}\right), \alpha \in R$, is a Chevalley system of $(\mathfrak{g}, \mathfrak{h})$ having the required properties.

Now we can prove our basic lemma.

LEMMA 3. Let $(\mathfrak{g}, H / 2)=\bigoplus \mathfrak{s}_{k}, k \in \mathbf{Z}$, be a simple flat complex Lie algebra and assume that the associated $\mathbf{Z}_{2}$-grading on $\mathfrak{g}$ coincides with $\mathfrak{g}=\mathfrak{k} \oplus \mathfrak{p}$. Then there exists $X \in \mathfrak{s}_{1}$ such that

$$
[X, \sigma(X)]=H .
$$

PROOF. Fix a Cartan subalgebra $\mathfrak{h}_{0}$ of $\mathfrak{k}_{0}$ such that $\mathfrak{h}_{0} \subset \mathfrak{s}_{0}$ and set $\mathfrak{h}=\mathfrak{h}_{0}+i \mathfrak{h}_{0}$. Let $\mathbf{R}$ be the root system of $(\mathfrak{g}, \mathfrak{h})$ and for $k \in \mathbf{Z}$ let

$$
R_{k}=\left\{\alpha \in R: \mathfrak{g}^{\alpha} \subset \mathfrak{s}_{k}\right\} .
$$

Also define

$$
R^{(0)}=\bigcup_{k \in \mathbf{Z}} R_{2 k}, \quad R^{(1)}=\bigcup_{k \in \mathbf{Z}} R_{2 k+1} .
$$

By Lemma 2 there exists a Chevalley system $\left(X_{\alpha}\right), \alpha \in R$, such that

$$
\sigma\left(X_{\alpha}\right)=(-1)^{k} X_{-\alpha}, \quad \alpha \in R^{(k)} .
$$

For $\alpha \in R_{1}$ let $Y_{\alpha}=-X_{-\alpha}$. Let $H_{\alpha}$ be the unique element of $\left[\mathfrak{g}^{\alpha}, \mathfrak{g}^{-\alpha}\right]$ such that $\alpha\left(H_{\alpha}\right)=2$. Recall that $\left[X_{\alpha}, X_{-\alpha}\right]=-H_{\alpha}, \alpha \in R$, and so $\left[X_{\alpha}, Y_{\alpha}\right]=H_{\alpha}$ for $\alpha \in R_{1}$.

We shall seek a solution of equation (1) in the form

$$
X=\sum_{\alpha \in R_{1}} \lambda_{\alpha} X_{\alpha}
$$

with all $\lambda_{\alpha}$ real. Then

$$
\sigma(X)=\sum_{\alpha \in R_{1}} \lambda_{\alpha} Y_{\alpha}
$$

and equation (1) can be written as

$$
\sum_{\alpha, \beta \in R_{1}} \lambda_{\alpha} \lambda_{\beta}\left[X_{\alpha}, Y_{\beta}\right]=H
$$


Assume first that our flat Lie algebra $(\mathfrak{g}, H / 2)$ is principal, i.e., that $R_{1}$ is a base, say $B$, of $R$. In that case $\alpha-\beta \notin R$ for $\alpha, \beta \in B$ and so (2) becomes

$$
\sum_{\alpha \in B} \lambda_{\alpha}^{2} H_{\alpha}=H
$$

Since $\alpha(H)=2$ for all $\alpha \in B$, this equation is equivalent to the system

$$
\sum_{\beta \in B} \alpha\left(H_{\beta}\right) \lambda_{\beta}^{2}=2, \quad \alpha \in B
$$

By a theorem of Vinberg $\left[\mathbf{1 0}\right.$, Theorem 3] the unique solution $\mu_{\beta}, \beta \in R$, of the system of linear equations

$$
\sum_{\beta \in B} \alpha\left(H_{\beta}\right) \mu_{\beta}=2, \quad \alpha \in B
$$

is positive in the sense that $\mu_{\beta}>0$ for each $\beta \in B$. It follows that the system (3) has a real solution.

Next assume that $(\mathfrak{g}, H / 2)$ is the simple flat Lie algebra $D_{n+m+1}\left(a_{m}\right), n>m \geq$ 1 , see [5 or 6]. In this case we shall use the notations for roots, the Chevalley system, etc., given in Bourbaki [3, Chapitre VIII, §13, no. 4, pp. 206-212]. Then $H / 2$ is the diagonal matrix of order $2 n+2 m+2$ whose diagonal entries are the integers $n, n-1, \ldots,-n$ and $m, m-1, \ldots,-m$ arranged in nonincreasing order. The set $R_{1}$ consists of the roots

$$
\begin{array}{lll}
\varepsilon_{i}-\varepsilon_{i+1}, \quad 1 \leq i \leq n-m ; \quad \varepsilon_{n-m}-\varepsilon_{n-m+2} ; & \\
\varepsilon_{n-m+2 k-1}-\varepsilon_{n-m+2 k+1}, & \varepsilon_{n-m+2 k}-\varepsilon_{n-m+2 k+1}, & 1 \leq k \leq m ; \\
\varepsilon_{n-m+2 k-1}-\varepsilon_{n-m+2 k+2}, & \varepsilon_{n-m+2 k}-\varepsilon_{n-m+2 k+2}, & 1 \leq k \leq m-1 ;
\end{array}
$$

and

$$
\varepsilon_{n+m-1}+\varepsilon_{n+m+1}, \quad \varepsilon_{n+m}+\varepsilon_{n+m+1} .
$$

In this case some of the $\lambda_{\alpha}$ can be taken to be zero. An explicit solution of equation (1) is provided by

$$
\begin{aligned}
X= & \lambda_{1} X_{\varepsilon_{1}-\varepsilon_{2}}+\lambda_{2} X_{\varepsilon_{2}-\varepsilon_{3}}+\cdots+\lambda_{n-m} X_{\varepsilon_{n-m}-\varepsilon_{n-m+1}} \\
& +\mu_{1} X_{\varepsilon_{n-m+1}-\varepsilon_{n-m+3}}+\nu_{1} X_{\varepsilon_{n-m+2}-\varepsilon_{n-m+4}} \\
& +\mu_{2} X_{\varepsilon_{n-m+3}-\varepsilon_{n-m+5}}+\nu_{2} X_{\varepsilon_{n-m+4}-\varepsilon_{n-m+6}} \\
& +\cdots \\
& +\mu_{m-1} X_{\varepsilon_{n+m-3}-\varepsilon_{n+m-1}}+\nu_{m-1} X_{\varepsilon_{n+m-2}-\varepsilon_{n+m}} \\
& +\rho_{1} X_{\varepsilon_{n+m-1}-\varepsilon_{n+m+1}}+\sigma_{1} X_{\varepsilon_{n+m-1}-\varepsilon_{n+m+1}} \\
& +\rho_{2} X_{\varepsilon_{n+m}-\varepsilon_{n+m+1}}+\sigma_{2} X_{\varepsilon_{n+m}+\varepsilon_{n+m+1}}
\end{aligned}
$$

where

$$
\begin{aligned}
\lambda_{k}^{2} & =k(2 n-k+1), \quad 1 \leq k \leq n-m ; \\
\mu_{k}^{2} & =(n-m)(n+m+1)+k(2 m-k+1), \quad 1 \leq k \leq m-1 ; \\
\nu_{k}^{2} & =k(2 m-k+1), \quad 1 \leq k \leq m-1 ;
\end{aligned}
$$

and $\rho_{1}+i \rho_{2}= \pm \sqrt{z}, \sigma_{1}+i \sigma_{2}= \pm \sqrt{w}$ with $z$ and $w$ complex numbers satisfying $z+w=(n-m)(n+m+1),|z|=|w|=m^{2}-3 m+4+\frac{1}{2}(n-m)(n+m+1)$. 
We omit the routine details of the verification of this claim.

There remain five exceptional simple flat Lie algebras to be dealt with, namely, $E_{8}\left(a_{1}\right), E_{8}\left(a_{2}\right), E_{7}\left(a_{1}\right), E_{7}\left(a_{2}\right)$, and $E_{6}\left(a_{1}\right)$. In order to exhibit an explicit solution of (1) it will be convenient to use the Chevalley system of $E_{8}$ constructed in our paper [4]. For the convenience of the reader we shall review some basic facts about this Chevalley system.

The Lie algebra $E_{8}$ is realized as Z-graded algebra $\mathfrak{g}=\bigoplus_{k \in \mathbf{Z}} \mathfrak{s}_{k}$ where $\mathfrak{s}_{0}=$ $V \otimes V^{*}, \mathfrak{s}_{1}=\bigwedge^{3} V, \mathfrak{s}_{-1}=\bigwedge^{3} V^{*}, \mathfrak{s}_{2}=\bigwedge^{2} V, \mathfrak{s}_{-2}=\bigwedge^{2} V^{*}, \mathfrak{s}_{3}=V, \mathfrak{s}_{4}=V^{*}$ and $\mathfrak{s}_{k}=0$ otherwise. Here $V$ denotes a complex vector space of dimension 8 with a fixed basis $e_{k}, 1 \leq k \leq 8$, and $V^{*}$ its dual space with the dual basis $e^{k}, 1 \leq k \leq 8$.

For the definition of the Lie bracket see [4]. We mention only that $\mathfrak{s}_{0} \cong \mathfrak{g l}(V)$, that the action of $\mathfrak{s}_{0}$ on each $\mathfrak{s}_{k}$ is the standard one, and that

$$
[a \wedge b \wedge c, f \wedge g \wedge h]=-\left|\begin{array}{cccc}
f(a) & f(b) & f(c) & f \\
g(a) & g(b) & g(c) & g \\
h(a) & h(b) & h(c) & h \\
a & b & c & 1 / 3
\end{array}\right|
$$

where $a, b, c \in V, f, g, h \in V^{*}$ and when evaluating this determinant the product of, say, $a$ and $f$ should be written as $a \otimes f$.

Using the formula (4) one finds that

$$
\left[e_{i j k}, e^{r j k}\right]=e_{i}^{r}
$$

if $i \neq r, i \stackrel{<}{<} j<k$, and $r<j$.

Writing $e_{i}^{j}=e_{i} \otimes e^{j}$, the subspace $\mathfrak{h}$ spanned by the elements $e_{i}^{i}, 1 \leq i \leq 8$, is a Cartan subalgebra of $\mathfrak{s}_{0}$ and of $\mathfrak{g}$. The Chevalley system of $(\mathfrak{g}, \mathfrak{h})$ is given by the elements:

$$
\begin{array}{ll}
e_{i}^{j},-e_{j}^{i} & (1 \leq i<j \leq 8) ; \\
e_{i},-e^{i} & (1 \leq i \leq 8) ; \\
e_{i j k},-e^{i j k} & (1 \leq i<j<k \leq 8) ; \\
e_{i j}, e^{i j} & (1 \leq i<j \leq 8) ;
\end{array}
$$

where $e_{i j k}=e_{i} \wedge e_{j} \wedge e_{k}, e^{i j k}=e^{i} \wedge e^{j} \wedge e^{k}$, etc.

In [4] $\lambda_{i}, 1 \leq i \leq 8$, is a basis of $\mathfrak{h}^{*}$ dual to the basis $e_{i}^{i}, 1 \leq i \leq 8$, of $\mathfrak{h}$. A base $B$ of the root system $R$ of $(\mathfrak{g}, \mathfrak{h})$ consists of the roots $\lambda_{i}-\lambda_{i+1}, 1 \leq i \leq 7$, and the $\operatorname{root} \lambda_{6}+\lambda_{7}+\lambda_{8}$ :

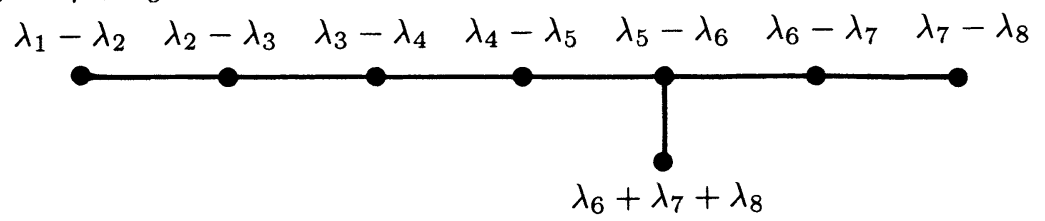

For $\alpha \in B$ the elements $H_{\alpha} \in \mathfrak{h}$ are given by

$$
\begin{aligned}
& h_{i}:=H_{\lambda_{i}-\lambda_{i+1}}=e_{i}^{i}-e_{i+1}^{i+1}, \quad 1 \leq i \leq 7, \\
& h_{8}:=H_{\lambda_{6}+\lambda_{7}+\lambda_{8}}=-\frac{1}{3}+e_{6}^{6}+e_{7}^{7}+e_{8}^{8},
\end{aligned}
$$

whence $-\frac{1}{3}$ means $-\frac{1}{3} \cdot \sum_{i=1}^{8} e_{i}^{i}$.

Case $E_{8}\left(a_{1}\right)$. In this case we have

$$
H=46 h_{1}+90 h_{2}+132 h_{3}+172 h_{4}+210 h_{5}+142 h_{6}+72 h_{7}+106 h_{8},
$$


and $R_{1}$ consists of the roots $\lambda_{i}-\lambda_{i+1}, i \neq 5, \lambda_{6}+\lambda_{7}+\lambda_{8}, \lambda_{4}-\lambda_{6}, \lambda_{5}-\lambda_{7}$, and $\lambda_{5}+\lambda_{7}+\lambda_{8}$.

An explicit solution of (1) is given by

$$
X=\sqrt{46} e_{1}^{2}+\sqrt{90} e_{2}^{3}+\sqrt{132} e_{3}^{4}+\rho_{1} e_{4}^{5}+\sigma_{1} e_{6}^{7}+\sqrt{72} e_{7}^{8}+\sqrt{106} e_{678}+\rho_{2} e_{4}^{6}+\sigma_{2} e_{5}^{7}
$$

where $\rho_{1}+i \rho_{2}= \pm \sqrt{z}, \sigma_{1}-i \sigma_{2}= \pm \sqrt{w}$ and $z$ and $w$ are complex numbers such that $|z|=172,|w|=142, z+w=-106$.

Case $E_{8}\left(a_{2}\right)$. We have

$$
H=38 h_{1}+74 h_{2}+108 h_{3}+142 h_{4}+174 h_{5}+118 h_{6}+60 h_{7}+88 h_{8},
$$

$R_{1}$ consists of the roots $\lambda_{i}-\lambda_{i+1}, i \neq 3,5 ; \lambda_{6}+\lambda_{7}+\lambda_{8}, \lambda_{2}-\lambda_{4}, \lambda_{3}-\lambda_{5}, \lambda_{4}-\lambda_{6}$, $\lambda_{5}-\lambda_{7}$, and $\lambda_{5}+\lambda_{7}+\lambda_{8}$. A solution of (1) is provided by

$$
X=\sqrt{38} e_{1}^{2}+\sqrt{74} e_{2}^{3}+\sqrt{34} e_{4}^{5}+\rho_{1} e_{6}^{7}+\sqrt{60} e_{7}^{8}+\sigma_{1} e_{678}+\sqrt{108} e_{3}^{6}+\rho_{2} e_{5}^{7}+\sigma_{2} e_{578}
$$

where $\rho_{1}+i \rho_{2}= \pm \sqrt{z}, \sigma_{1}+i \sigma_{2}= \pm \sqrt{w}$ and $z$ and $w$ are complex numbers such that $|z|=118,|w|=88, z+w=74$.

Formula (5) is useful when one checks that $X$ is indeed a solution.

Case $E_{7}\left(a_{1}\right)$. We have

$$
H=21 h_{2}+40 h_{3}+57 h_{4}+72 h_{5}+50 h_{6}+26 h_{7}+37 h_{8},
$$

and $R_{1}$ is the same as in case $E_{8}\left(a_{1}\right)$ except that the root $\lambda_{1}-\lambda_{2}$ should be omitted. A solution $X$ of (1) is given by

$$
X=\sqrt{21} e_{2}^{3}+\sqrt{40} e_{3}^{4}+\sigma_{1} e_{4}^{5}+\rho_{1} e_{6}^{7}+\sqrt{26} e_{7}^{8}+\sqrt{37} e_{678}+\sigma_{2} e_{4}^{6}+\rho_{2} e_{5}^{7},
$$

where $\rho_{1}+i \rho_{2}= \pm \sqrt{z}, \sigma_{1}-i \sigma_{2}= \pm \sqrt{w}$ and $z$ and $w$ are complex numbers satisfying $|z|=50,|w|=57, z+w=-37$.

Case $E_{7}\left(a_{2}\right)$. Now

$$
H=17 h_{2}+32 h_{3}+47 h_{4}+60 h_{5}+42 h_{6}+22 h_{7}+31 h_{8}
$$

and $R_{1}$ is the same as in the case $E_{8}\left(a_{2}\right)$ except that the root $\lambda_{1}-\lambda_{2}$ should be omitted. A solution $X$ of (1) is given by

$$
X=\sqrt{17} e_{2}^{3}+\sqrt{15} e_{4}^{5}+\rho_{1} e_{6}^{7}+\sqrt{22} e_{7}^{8}+\sigma_{1} e_{678}+\sqrt{32} e_{3}^{6}+\rho_{2} e_{5}^{7}+\sigma_{2} e_{578},
$$

where $\rho_{1}+i \rho_{2}= \pm \sqrt{z}, \sigma_{1}+i \sigma_{2}= \pm \sqrt{w}$ and $z$ and $w$ are complex numbers satisfying $|z|=42,|w|=31, z+w=17$.

Case $E_{6}\left(a_{1}\right)$. In this case

$$
H=12 h_{3}+22 h_{4}+30 h_{5}+22 h_{6}+12 h_{7}+16 h_{8},
$$

and $R_{1}$ is the same as in case $E_{8}\left(a_{1}\right)$ except that the roots $\lambda_{1}-\lambda_{2}$ and $\lambda_{2}-\lambda_{3}$ should be omitted. A solution $X$ of (1) is given by

$$
X=\sqrt{12} e_{3}^{4}+\rho_{1} e_{4}^{5}+\sigma_{1} e_{6}^{7}+\sqrt{12} e_{7}^{8}+4 e_{678}+\rho_{2} e_{4}^{6}+\sigma_{2} e_{5}^{7},
$$

where $\rho_{1}+i \rho_{2}= \pm \sqrt{z}, \sigma_{1}-i \sigma_{2}= \pm \sqrt{w}$, and $z$ and $w$ are complex numbers satisfying $|z|=|w|=22, z+w=-16$. This completes the proof of the lemma. 
5. Proof that $\psi$ is surjective. Let $E$ be a nonzero nilpotent element in $\mathfrak{p}$. We have to show that there exists a complex Cayley triple $(X, H, Y)$ such that $X \in K \cdot E$. The proof is by induction on the dimension of $\mathfrak{g}$.

We can embed $E$ in a normal $\mathfrak{s l}_{2}$-triple $(E, H, F)$. Since $H$ is a real semisimple element it is $K$-conjugate to an element of $i \mathfrak{k}_{0}$. Hence by replacing this triple by a suitable $K$-conjugate we may assume that $H \in i \mathfrak{k}_{0}$.

Let $\mathfrak{s}=\bigoplus \mathfrak{s}_{k}, k \in \mathbf{Z}$, be the $\mathbf{Z}$-graded subalgebra of the $\mathbf{Z}_{2}$-graded algebra $\mathfrak{g}=\mathfrak{k}+\mathfrak{p}$ defined as follows:

$$
\mathfrak{s}_{k}=\{X \in \mathfrak{k}:[H, X]=2 k X\}
$$

for $k$ even and

$$
\mathfrak{s}_{k}=\{X \in \mathfrak{p}:[H, X]=2 k X\}
$$

for $k$ odd. Clearly $E \in \mathfrak{s}_{1}$ and by a result of Vinberg [12, Lemma 2] $\mathfrak{s}$ is reductive. If $\mathfrak{s} \neq \mathfrak{g}$ then the induction hypothesis can be applied to the associated $\mathbf{Z}_{2}$-graded algebra $\mathfrak{s}=\mathfrak{s} \cap \mathfrak{k} \oplus \mathfrak{s} \cap \mathfrak{p}$ and the element $E$.

Hence we may assume that $\mathfrak{s}=\mathfrak{g}$. Since the centralizer of $H$ in $\mathfrak{s}$ is $\mathfrak{s}_{0}, \mathfrak{s}=\mathfrak{g}$, and $\mathfrak{s}_{0} \subset \mathfrak{k}$, it follows that rank $\mathfrak{k}=$ rank $\mathfrak{g}$. Let us fix a Cartan subalgebra $\mathfrak{h}_{0}$ of $\mathfrak{k}_{0}$ such that $i H \in \mathfrak{h}_{0}$. Set $\mathfrak{h}=\mathfrak{h}_{0}+i \mathfrak{h}_{0}$.

By a theorem of Vinberg and Elašvili $[\mathbf{1 3}$, p. 223] there exist $X \in K \cdot E$ and a regular semisimple subalgebra $\mathfrak{t}$ of $\mathfrak{g}$ normalized by $\mathfrak{h}$ such that $X \in \mathfrak{t}$ and $X$ is a semiregular nilpotent element of $t$.

Since $\mathfrak{h}$ normalizes $\mathfrak{t}$, it follows that the $\mathbf{Z}_{2}$-grading $\mathfrak{g}=\mathfrak{k}+\mathfrak{p}$ induces a $\mathbf{Z}_{2^{-}}$ grading on $\mathfrak{t}$, i.e., that $\mathfrak{t}=\mathfrak{t} \cap \mathfrak{k} \oplus \mathfrak{t} \cap \mathfrak{p}$. If $\mathfrak{t} \neq \mathfrak{g}$ then the inductive hypothesis can be applied to $\mathfrak{t}$ and $X$. Hence we may assume that $\mathfrak{t}=\mathfrak{g}$, i.e., that $E$ is a semiregular nilpotent element of $\mathfrak{g}$.

By Lemma 1 the Z-graded algebra $\mathfrak{g}=\mathfrak{s}=\bigoplus \mathfrak{s}_{k}$ is flat and $E$ is a generic element of $\mathfrak{s}_{1}$. Since $H \in i \mathfrak{h}_{0} \subset i \mathfrak{k}_{0}$, we have $\sigma(H)=-H$ and consequently $\sigma\left(\mathfrak{s}_{k}\right)=\mathfrak{s}_{-k}$ for all $k$.

Assume that there is an $X \in \mathfrak{s}_{1}$ such that $[X, \sigma(X)]=H$. Then $(X, H,-\sigma(X))$ is a complex Cayley triple and by a result of Kostant and Rallis [8, Lemma 4] the normal $\mathfrak{s l}_{2}$-triples $(E, H, F)$ and $(X, H,-\sigma(X))$ are $K$-conjugate.

Hence it suffices to prove the existence of an element $X \in \mathfrak{s}_{1}$ such that $[X, \sigma(X)]$ $=H$. Since every flat Lie algebra is a direct product of simple flat Lie algebras we have

$$
(\mathfrak{g}, H / 2)=\left(\mathfrak{g}^{(1)}, H_{1} / 2\right) \times \cdots \times\left(\mathfrak{g}^{(m)}, H_{m} / 2\right)
$$

where each $\left(\mathfrak{g}^{(k)}, H_{k} / 2\right)$ is a simple flat Lie algebra and $H=H_{1}+\cdots+H_{m}$. This shows that without any loss of generality we may now assume that $\mathfrak{g}$ is simple.

In Lemma 3 we have shown that in the case of simple flat Lie algebras the equation $[X, \sigma(X)]=H$ indeed has a solution for $X$. This completes the proof of the conjecture.

Addendum (February 1987). The maps $\phi$ and $\psi_{0}$ defined in $\S 3$ are in fact bijective. In view of the results mentioned there and our main theorem, the claim follows from the following proposition.

PROPOSITION. The map $\phi$ is injective.

Proof. Let $(E, H, F)$ and $\left(E^{\prime}, H^{\prime}, F^{\prime}\right)$ be two real Cayley triples with $E^{\prime} \in$ $G_{0} \cdot E$. By $\left[3\right.$, Chapter VIII, $\S 11$, Lemma 4] these triples are $G_{0}$-conjugate. By 
using [9, Proposition 1.1] it follows that $E^{\prime}-F^{\prime} \in K_{0} \cdot(E-F)$. Hence we may assume that $E^{\prime}-F^{\prime}=E-F=Z$, say. Let $G_{0}^{Z}$ (resp., $K_{0}^{Z}$ ) be the centralizer of $Z$ in $G_{0}$ (resp., $K_{0}$ ). Fix a maximal compact subgroup $M$ of $G_{0}^{Z}$ containing $K_{0}^{Z}$. If $x \in M$ write $x=y \exp (X)$ with $y \in K_{0}$ and $X \in \mathfrak{p}_{0}$. By using an argument of L. Preiss-Rothschild [9, Proof of Proposition 1.1] it follows from $\exp (X) \cdot Z=y^{-1} \cdot Z$ that $y^{-1} \cdot Z=Z$. Hence $y \in K_{0}^{Z}, \exp (X) \in M$ and since $M$ is compact we must have $X=0$. Thus $M=K_{0}^{Z}$.

By [8, p. 779] $\mathfrak{g}_{0}^{Z}=\mathfrak{k}_{0}^{Z} \oplus \mathfrak{p}_{0}^{Z}$ is a Cartan decomposition of $\mathfrak{g}_{0}^{Z}$ and consequently $G_{0}^{Z}=K_{0}^{Z} \cdot \exp \left(\mathfrak{p}_{0}^{Z}\right)$.

If $a \in G_{0}$ is an element which maps the triple $(E, H, F)$ to $\left(E^{\prime}, H^{\prime}, F^{\prime}\right)$ then $a \in G_{0}^{Z}$ and $a \cdot(E+F)=E^{\prime}+F^{\prime}$. Write $a=b \exp (Y)$ with $b \in K_{0}^{Z}$ and $Y \in \mathfrak{p}_{0}^{Z}$. Then by applying the above mentioned argument to $\exp (Y) \cdot(E+F)=b^{-1} \cdot\left(E^{\prime}+F^{\prime}\right)$ we infer that $b^{-1} \cdot\left(E^{\prime}+F^{\prime}\right)=E+F$. Thus $b \in K_{0}$ sends $(E, H, F)$ to $\left(E^{\prime}, F^{\prime}, H^{\prime}\right)$.

ADDED IN PROOF. After this paper was written D. King informed me that Jiro Sekiguchi had also proved Kostant's conjecture (by a different method) in a preprint entitled Remarks on real nilpotent orbits of a symmetric pair.

\section{REFERENCES}

1. L. V. Antonyan, Classification of four-vectors of the eight-dimensional space, Trudy Sem. Vektor. Tenzor. Anal. no. 20, Moscow University, 1981, pp. 144-161.

2. D. Barbasch, Fourier inversion for unipotent invariant integrals, Trans. Amer. Math. Soc. 249 (1979), 51-83.

3. N. Bourbaki, Groupes et algèbres de Lie, Chapitres VII et VIII, Hermann, Paris, 1975.

4. D. Ž. Djokovic, Classification of trivectors of an eight-dimensional real vector space, Linear and Multilinear Algebra 13 (1983), 3-39.

5. E. B. Dynkin, Semisimple subalgebras of semisimple Lie algebras, Mat. Sb. 30 (1953), 349-462; English transl., Amer. Math. Soc. Transl. (2) 6 (1957), 111-245.

6. G. B. Elkington, Centralizers of unipotent elements in semisimple algebraic groups, J. Algebra 23 (1972), 137-163.

7. D. R. King, The Cayley transform of a nilpotent conjugacy class in a real semi-simple Lie algebra (preprint).

8. B. Kostant and S. Rallis, Orbits and representations associated with symmetric spaces, Amer. J. Math. 93 (1971), 753-809.

9. L. Preiss-Rothschild, Orbits in a real reductive Lie algebra, Trans. Amer. Math. Soc. 168 (1972), 403-421.

10. E. B. Vinberg, Discrete linear groups generated by reflections, Izv. Akad. Nauk SSSR Ser. Mat. 35 (1971), 1072-1112.

11. _ On the classification of the nilpotent elements of graded Lie algebras, Dokl. Akad. Nauk SSSR 225 (1975), 745-748=Soviet Math. Dokl. 16 (1975), 1517-1520.

12. Classification of the homogeneous nilpotent elements of a semisimple graded Lie algebra, Trudy Sem. Vektor. Tenzor. Anal., no. 19, Moscow University, 1979, pp. 155-177.

13. E. B. Vinberg and A. G. Elašvili, Classification of tri-vectors of the 9-dimensional space, Trudy Sem. Vektor. Tenzor. Anal., no. 18, Moscow University, 1978, pp. 197-233.

Department of Pure Mathematics, University of Waterloo, Waterloo, OnTARIO, CANADA N2L 3G1 